\title{
Die chemische Behandlung physiologischer Probleme
}

Vorwiegend gezeigt anhand von Berner Autoren

Von William Arthur Liebi

\section{Einführung}

Erst, nachdem die Chemie durch neue theoretische Vorstellungen und experimentelle Forschung eine tragende Grundlage erhalten hatte, konnten leistungsfähige Methoden entwickelt werden, um tierische Materialien Fluida und Solida - chemisch zu untersuchen. Dieser Zustand wurde um 1840 erreicht, als zugleich auch die naturphilosophische Strömung, die in Deutschland zwischen 1800 und 1820 dominierte, überwunden war. Im Jahrzehnt nach 1840 erschienen zahlreiche Vorschläge zur chemischen Untersuchung tierischer Materialien mit zum Teil ausführlichen Methodenbeschreibungen.

Es zeigten sich auch methodische Schwierigkeiten: Das Probevolumen für Blutuntersuchungen lag relativ hoch. Nach 1830 verzichtete man zunehmend auf den Aderlaß, was bewirkte, daß Blut nicht mehr ohne weiteres verfügbar war. Der Blutentnahme zu Untersuchungszwecken standen die Embolie- und Infektionsgefahr entgegen. Erst die Erfindung der Venenpunktionsnadel 1905 sowie die Entwicklung von Mikromethoden ab 1914 verbesserten die Situation.

Die Aufgliederung der Wissenschaft in Disziplinen ist kennzeichnend für das 19. Jahrhundert. Disziplinen entstehen vielfach dadurch, daß sich neue Forschungsfelder herausbilden. Leitlinien zur Begründung von Forschungsbereichen nennen wir «Konzepte».

Innerhalb des Rahmenthemas «Die Entwicklung der Physiologie im 19. Jahrhundert und die Schweiz» soll gezeigt werden, in was für Konzepten chemische Denkweisen, Methoden oder Forschungsresultate herangezogen wurden, um physiologische Fragestellungen zu beantworten. Im Sinne einer lokalen Einschränkung besprechen wir Konzepte von Autoren, die in Bern tätig waren. Um diese Berner Konzepte medizin- und chemiehistorisch etwas einzuordnen, werden auch Konzepte von auswärtigen, international bekannten Autoren angeführt. Werden keine besonderen Ortsangaben gemacht, handelt es sich jeweils um Berner Autoren. Deren Konzepte 
werden in chronologischer Reihenfolge dargestellt. Als Überschriften stehen die Bezeichnungen jener Disziplinen, innerhalb derer die Konzepte angewandt wurden.

\section{Naturphilosophische Anthropologie}

An der Schwelle zum 19. Jahrhundert erschien vom Philosophieprofessor Johannes Samuel Ith (1747-1813) ${ }^{1}$ ein Werk, in dem die «Philosophie des Menschen physiologisch betrachtet» die Anthropologie im engsten Sinne bedeutet $^{2}$. Ith berücksichtigte die Ergebnisse der Anatomie, Physiologie ${ }^{3}$ und Chemie ${ }^{4}$, um die sechs verschiedenen Kräfte ${ }^{5}$, welche seiner Auffassung nach im menschlichen Organismus wirken, naturphilosophisch besser bestimmen zu können. Aus dieser Perspektive heraus kritisierte Ith 1894, daß man Blutproben bisher nur mikroskopisch untersucht oder nur deren Veränderungen nach Stehenlassen festgestellt habe; nur unlängst sei begonnen worden, das Blut chemisch zu analysieren ${ }^{6}$.

Iths Beispiel stützt die These Friedrich von Engelhardts, wonach sich Naturphilosophie und Naturwissenschaften nicht ausschließen, sondern gegenseitig bedingen und ergänzen ${ }^{\text {. }}$.

Im Gegensatz zu Ith versprach sich der Schelling-Schüler und spätere Berner Philosophieprofessor Ignaz Paul Vital Troxler $(1780-1866)^{8}$ von der Chemie keine Aufschlüsse. Troxler schrieb 1803:

«Überhaupt ist uns ja die Chemie noch immer jene Elemente, aus denen sie ihre Welt baut, schuldig geblieben, und wird es auch wohl immer, da alle ihre Stoffe nur chimairische Wesen sind, und nicht anders als wechselnde Formen ein und derselben Materien.. ${ }^{9}$

\section{Tierchemie}

1831 schlug der Chemieprofessor Carl Emanuel Brunner (1796-1867) ${ }^{10}$ vor, daß Anatomen, Physiologen und Chemiker zusammenarbeiten sollten, um die Chemie der tierischen Substanzen hinsichtlich der Medizin zu betreiben ${ }^{11}$, Brunner, der sich methodisch auf Lavoisier beruft ${ }^{12}$, hatte sich studienhalber auch in Paris aufgehalten ${ }^{13}$.

Der Physiologie fällt nach Brunner auch die Aufgabe zu, die Wirkungen der Lebenskraft zu studieren. Dabei ist die Physiologie auf die Chemie angewiesen, denn: 
«Nur sie kann die Stoffe, welche die Lebenskraft verarbeitet und die, welche sie schafft, miteinander vergleichen.» ${ }^{14}$

Die Lebenskraft selber scheine dem Untersucher verborgen zu bleiben ${ }^{15}$.

Für oder gegen die Einführung einer Lebenskraft äußerten sich viele Experimentatoren des 19. Jahrhunderts.

Brunner war von Jöns Jacob Berzelius (1779-1848) als Analytiker sehr geschätzt ${ }^{16}$. Sein Konzept einer medizinorientierten organischen Chemie erwähnte Brunner etwa zehn Jahre vor dem Erscheinen von Justus Liebigs (1803-1873) viel diskutierter Monographie «Die organische Chemie in ihrer Anwendung auf Physiologie und Pathologie» ${ }^{17}$.

\section{Anatomie}

Für Friedrich Andreas Gerber (1797-1872) ${ }^{18}$, Anatomieprofessor an der Tierarzneischule, gründet die Physiologie auf der allgemeinen Anatomie. Nach Gerbers Auffassung von 1840 sollte besonders die Mikroskopie als anatomische Hilfsmethodik entwickelt werden ${ }^{19}$. Die Zoochemie ist ebenfalls eine Hilfswissenschaft der Anatomie, indem nach einer mechanischen Auftrennung die Bestandteile des Organismus chemisch analysiert wer$\operatorname{den}^{20}$.

Der Anatome Christoph Theodor Aeby (1835-1885) ${ }^{21}$ führte 1871 aus, wie er die physiologische und morphologische Bedeutung der menschlichen Organe zu begründen gedenkt. Bevor die Einheit des Organismus funktionell-anatomisch erklärt werden kann, muß dieser aufgetrennt werden. Über die gewöhnlichen anatomischen Instrumente hinaus läßt Aeby den Untersucher auch chemische Methoden einsetzen:

«Mit welchen Mitteln er sein Ziel zu erreichen sucht, ... ob er mit Messer und Scheere [sic!) oder mit chemischen oder optischen Vorrichtungen in seinen Gegenstand eindringt, ist ohne wesentliche Bedeutung.. ${ }^{22}$

Aeby wie Gerber stehen in der Denktradition des in Göttingen berühmt gewordenen Albrecht von Hallers (1708-1777). Dieser erklärte gegen 1760, daß zum physiologischen Arbeiten der Körperbau bekannt sein müsse und daher die Physiologie mit der Anatomie verbunden sei ${ }^{23}$. Für Haller war die Scheidekunst «eine Gattung von der Zergliederungskunst»» ${ }^{24}$. Um die 
Beschaffenheit von Körperflüssigkeiten zu ermitteln, setzte Haller Fäulnis, Feuer und Flüssigkeiten bekannter Wirkung ein ${ }^{25}$.

\section{Physiologische Pathologie}

1843 äußerte sich der Polikliniker Emanuel Eduard Fueter (1801-1855) ${ }^{26}$, daß aus der gegenseitigen Durchdringung von Physiologie und Pathologie künftig eine wissenschaftlich begründete physiologische Pathologie hervorgehen könnte. Idealerweise sollten die Erscheinungen am einzelnen Kranken gesetzmäßig erklärbar werden ${ }^{27}$. Solange dies nicht möglich sei, müßten die Verhältnisse noch speziell-pathologisch formuliert werden ${ }^{28}$. Zur Überführung der Pathologie in eine physiologische Pathologie erhofft sich Fueter besonders durch die mikroskopische und chemische Methodik viele Einsichten:

«... was sind wir nicht berechtigt vom Mikroskop, von der Chemie in den nächsten Zeiten zu erwarten?» ${ }^{29}$

Viel von der Chemie erwarteten auch der Wiener pathologische Anatome Carl Rokitansky (1804-1878) und der Berliner Pathologe Rudolf Virchow (1821-1902). Die neue Disziplin im Schnittbereich von Physiologie und Pathologie wollten die Autoren unter Verwendung chemischer Methoden von ihren eigenen Fächern aus begründen ${ }^{30}$.

Der Frerichs-Schüler und spätere medizinische Kliniker Bernhard Naunyn (1839-1925) war 1871/72 in Bern, kann also nur am Rande als Berner Autor gelten. Nach Naunyn will man in der pathologischen Physiologie die Krankheitserscheinungen so weitgehend wie möglich auf normale physiologische Zustände zurückführen. Die Physiologie selber stützt sich auf die Anatomie und die Chemie $a b^{31}$.

\section{Physiologie}

Nach Gabriel Gustav Valentin (1810-1883) ${ }^{32}$, Professor der Anatomie und Physiologie, einem ehemaligen Schüler Purkynes in Breslau, befaßt sich die Humanphysiologie mit den materiell-psychischen Funktionen des lebenden Organismus $^{33}$. 1844 erwähnte Valentin drei Methodenrichtungen der Physiologie: 
1. Die anatomische, physikalische und chemische Untersuchung. Hierbei lassen sich die Ergebnisse von Versuchsreihen, die am Leichnam, an Organpräparaten oder im Reagenzglas durchgeführt wurden, auf den lebenden Organismus übertragen ${ }^{34}$.

2. Die physiologische Beobachtung und das physiologische Experiment an Tieren ${ }^{35}$.

3. Das Studium krankhafter Zustände ${ }^{36}$.

Jan Evangelista Purkyne (1781-1869) trug die Physiologie ab 1827 nach ähnlichen Gesichtspunkten vor, ohne jedoch Krankheitszustände zu beach$\operatorname{ten}^{37}$.

\section{Urinanalyse}

Nach einem Kursbesuch bei Johann Florian Heller (1813-1871) in Wien ${ }^{38}$ publizierte der spätere Privatdozent und Oberfeldarzt Franz Adolf Ziegler (1833-1899) ${ }^{39}$ im Jahre 1861 seine Dissertation mit dem Titel «Die Uroscopie am Krankenbette» ${ }^{40}$.

Der praktische Arzt soll zur Diagnostik über einfache harnchemische Methoden verfügen können ${ }^{41}$. Aufwendige Methoden sind nach Ziegler dann berechtigt, wenn ein Krankheitsvorgang physiologisch erklärt werden soll ${ }^{42}$.

Mit dem gleichen Doppelziel wollten 1894 die Pariser Pharmazeuten Antoine-Augustin Parmentier (1737-1813) und Nicolas Déyeux (17451837) Urin untersuchen; sie gaben jedoch keine methodischen Anleitungen ${ }^{43}$.

\section{Medizinische und physiologische Chemie}

Marceli Nencki (1847-1901) ${ }^{44}$, Schüler Adolf von Baeyers (1835-1917) in Berlin, kam durch Vermittlung von Naunyn nach Bern ${ }^{45}$, wo er sich von 1872 bis 1891 vor allem als organischer und medizinischer Chemiker etablierte. Nencki äußerte sich erst im Jahre 1900 programmatisch ${ }^{46}$. Die Entfaltung der vorherigen Forschungsrichtungen können wir aus den veröffentlichten Arbeiten von Nencki und seiner Schüler erschließen ${ }^{47}$.

Nach 1878 hat Nencki keine für ihn grundsätzlich neuen Themen aufgegriffen. Systematisch betrachtet lassen sich Nenckis Forschungsrichtungen wie folgt bestimmen: 
- Organische Chemie als chemische Untersuchungen von Naturstoffen und anderen Kohlenstoffverbindungen.

- Medizinische Chemie mit den Teildisziplinen:

1. Physiologische Chemie als Metabolismus körpereigener Stoffe.

2. Pathologische Chemie als Metabolismus bei Krankheitszuständen eines Organismus.

- Chemische Richtung der Pharmakologie als Metabolismus körperfremder Substanzen.

- Beiträge zu Einzellern und zur Infektabwehr.

Die Beiträge zur organischen und physiologischen Chemie ziehen sich durch Nenckis Schaffen hindurch. Pathologische Chemie und Pharmakologie wurden von ihm weniger intensiv bearbeitet. Nachdem Nencki 1888 einen zusätzlichen Lehrauftrag für Bakteriologie erhielt, ergaben sich regelmäßig Arbeiten aus diesem Bereich.

Der Arzt, Pharmazeut und Chemiker Antoine François Fourcroy (1755-1809) konzipierte 1800 die medizinische Chemie mit den Teilgebieten physiologische, pathologische, pharmakologische und hygienische Chemie $^{48}$. Die Tierchemie, die Fourcroy um 1790 konzipierte, gab die methodische Basis zur medizinischen Chemie ab.

Die chemischen Aspekte der Pharmakologie sowie der Hygiene gingen in den entsprechenden Disziplinen auf, während die physiologische und pathologische Chemie im Rahmen der medizinischen Chemie das ganze 19. Jahrhundert hindurch miteinander verbunden blieben. Die medizinische Chemie war je nachdem in eigenen Laboratorien in pathologischen Instituten oder in medizinischen Kliniken untergebracht ${ }^{49}$.

1842-52 erschien in Leipzig das dreibändige Lehrbuch von Carl Gotthelf Lehmann (1812-1863) zu einer vom medizinisch-chemischen Rahmen losgelösten physiologischen Chemie ${ }^{50}$. Diese war vor allem als chemische Richtung innerhalb der physiologischen Institute angesiedelt. Das 1846 speziell für physiologische Chemie in Tübingen eingerichtete Laboratorium gilt als erstes seiner Art ${ }^{51}$. 


\section{Anmerkungen}

Die vollständigen Titel der zitierten Werke sind im Literaturverzeichnis angegeben.

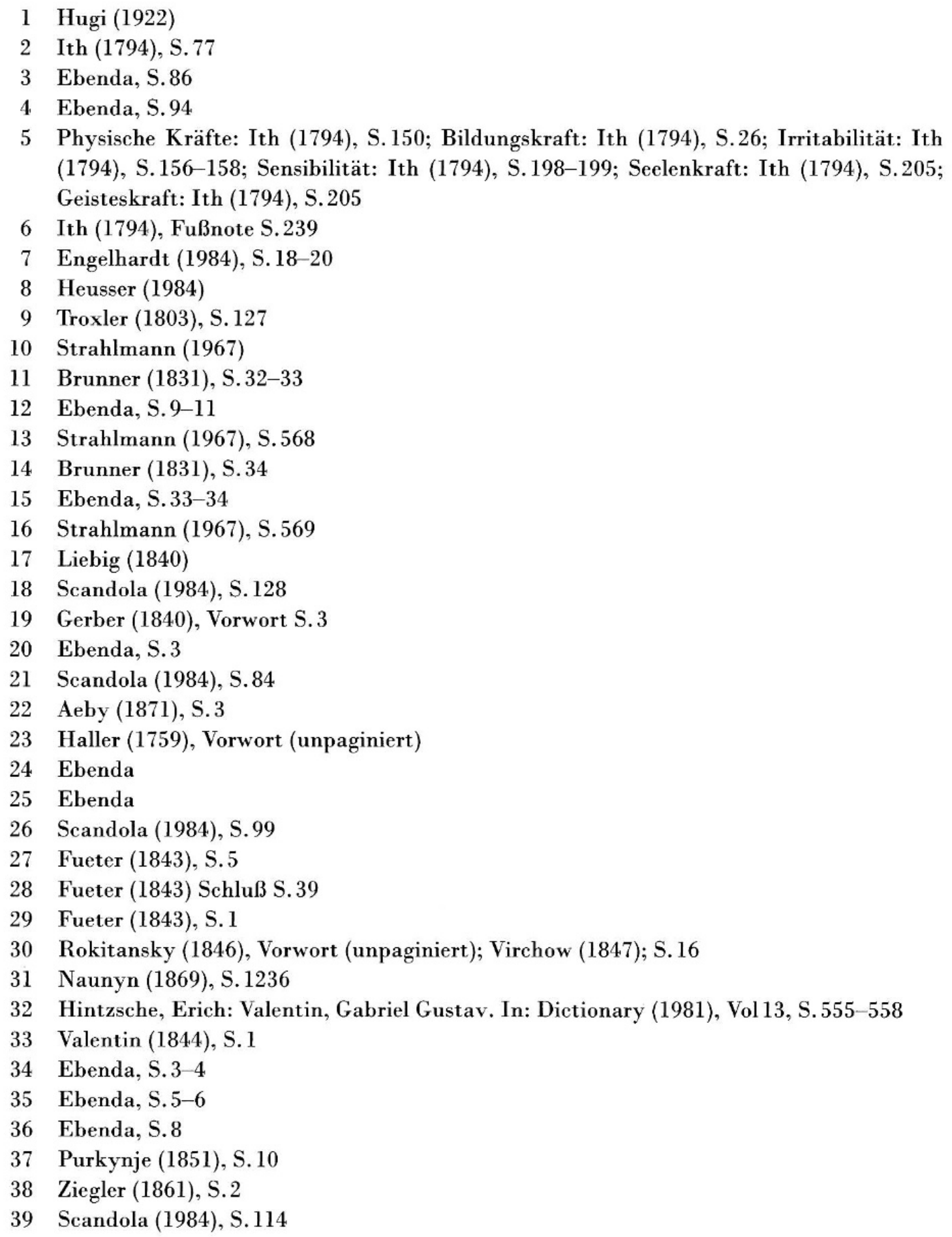


42 Ebenda, S. 1-2

43 Parmentier et Déyeux (1794), Fußnoten S. 389-390

44. Bickel (1972)

45 Naunyn (1925), S. 139-140 und 228

46 Nencki (1900). In: Nencki (1904), S. 719-727

47 Nencki (1904)

48 Premier tableau. Généralités de la chimie; chimie médicale. In: Fourcroy (1800) (unpaginiert)

49 Einzelheiten siehe Kohler (1982)

50 Lehmann (1842-52)

51 Hesse (1976), S. 18-19

\section{Literaturverzeichnis}

Aeby, Christoph: Der Bau des menschlichen Körpers mit besonderer Rücksicht auf seine morphologische und physiologische Bedeutung. Ein Lehrbuch der Anatomie für Ärzte und Studierende. Leipzig: Vogel, 1871

Bickel, Marcel H.: Marceli Nencki, 1847-1901. Bern: Huber, 1972

Brunner, Carl: Rede gehalten bei dem Antritte des Prorektorates an der Akademie zu Bern, den 24. Oktober 1831. Bern: Haller, 1831

Dictionary of scientific biography. Vol.1-16. New York: Scribner's Sons, 1981

Engelhardt, Dietrich von: Der metaphysische Krankheitsbegriff des deutschen Idealismus. Schellings und Hegels naturphilosophische Grundlegung. In: Seidler, Eduard (Hrsg.): Medizinische Anthropologie. Beiträge für eine Theoretische Pathologie. Berlin: Springer, 1984, S. 17-31

Fourcroy, Antoine François: Tableaux synoptiques de chimie, pour servir de résumé aux leçons données sur cette science dans les écoles de Paris. Paris: Baudoin, 1800

Fueter, Eduard: Über das naturgemäße Verhältniß der Heilkunst zur Krankheitslehre. In: Schweizerische Zeitschrift für Medicin, Chirurgie und Geburtshülfe. 1 (1843), S. 1-23

- Über das naturgemäße Verhältniß der Heilkunst zur Krankheitslehre. (Schluß.) In: Schweizerische Zeitschrift für Medicin, Chirurgie und Geburtshülfe. 2 (1843), S. 3-48

Gerber, Friedrich: Handbuch der allgemeinen Anatomie des Menschen und der Haussäugetiere, Größtentheils nach eigenen Untersuchungen und mit Benutzung der neuesten Entdeckungen im Gebiete dieser Wissenschaft. Bern: Dalp, 1840

Haller, Albrecht von: Anfangsgründe der Phisiologie des menschlichen Körpers. Band 1. Berlin/Leipzig: Voss, 1759

Hesse, Fritz: Professor Dr. med. et chir. Julius Eugen Schlossberger (1818-1860) - Begründer der physiologischen Chemie in Tübingen - Leben und Werk. Düsseldorf: Triltsch, 1976

Heusser, Peter: Der Schweizer Arzt und Philosoph Ignaz Paul Vital Troxler (1780-1866): Seine Philosophie, Anthropologie und Medizintheorie. Basel: Schwabe, 1984

Hugi, Walther: Professor Johann Samuel Ith von Bern 1747-1813. Beitrag zur bernischen Schul- und Gelehrtengeschichte. Diss. phil. Bern. Langensalza: Beyer und Söhne, 1922 
Ith, Johann: Versuch einer Anthropologie oder Philosophie des Menschen nach seinen körperlichen Anlagen. Teil 1. Bern: Haller, 1794

Kohler, Robert E.: From medical chemistry to biochemistry. The making of a biomedical discipline. Cambridge: Cambridge University Press, 1982

Lehmann, Carl Gotthelf: Lehrbuch der physiologischen Chemie. Bände 1-3. Leipzig: Engelmann, $1842-52$

Liebig, Justus: Die organische Chemie in ihrer Anwendung auf Agricultur und Physiologie. Braunschweig: Vieweg, 1840

Naunyn, Bernhard: Antrittsrede in Dorpat. Gehalten am 20.VIII./1.IX. 1869. In: Naunyn (1909), S. 1304-1311

- Gesammelte Abhandlungen. Band 2. Würzburg: Stürtz, 1909

- Erinnerungen, Gedanken und Meinungen. München: Bergmann, 1925

Nencki, Marceli: Über die Aufgaben der biologischen Chemie. Eröffnungsrede am IX. Congress polnischer Naturforscher und Ärzte in Krakau (1900). Deutsche Übersetzung. In: Nencki (1904), Band 2, S.719-727

- Marceli Nencki opera omnia. Gesammelte Arbeiten von Prof. M. Nencki. Bände 1-2. Braunschweig 1904

Parmentier, Antoine-Augustin, Déyeux, Nicolas: Mémoire sur le sang... (erster Teil). In: Journal de physique, de chimie et d'histoire naturelle ... Paris I (1794), S. 372-390.

Purkynje, Johann Evangelista: Über den Begriff der Physiologie... Rede gehalten bei der Eröffnung des physiologischen Institutes zu Prag am 6.Oktober 1851. o. O., o. J.

Rokitansky, Carl: Lehrbuch der allgemeinen pathologischen Anatomie. Band 1. Wien: Braunmüller und Seidel, 1846

Scandola, Pietro (Red.): Die Dozenten der bernischen Hochschule. Ergänzungsband zu: Hochschulgeschichte Berns 1528-1984. Zur 150-Jahr-Feier der Universität 1984. Bern: Universität Bern, 1984

Strahlmann, Berend: Carl Emanuel Brunner (1796-1867) und die Anfänge des chemischen Unterrichts an der Berner Hochschule. In: Chimia 21 (1967), S. 566-572

Troxler, Ignaz Paul Vital: Ideen zur Grundlage der Nosologie und Therapie. Jena: Academische Buchhandlung, 1803

Valentin, Gabriel Gustav: Lehrbuch der Physiologie des Menschen für Ärzte und Studierende. Band 1. Braunschweig: Vieweg, 1844

Virchow, Rudolf, Über die Standpunkte in der wissenschaftlichen Medicin. (Gelesen in der Jahressitzung der Gesellschaft für wissenschaftliche Medicin zu Berlin am 5.December 1846.) In: Archiv für pathologische Anatomie und Physiologie und für klinische Medicin. Band 1. Berlin: 1847, S.3-19

Ziegler, Adolf: Die Uroscopie am Krankenbette. Diss. med. Bern: Haller, 1861

\section{Summary}

During the 19th century, chemical thinking, methods or results were used to resolve physiological problems in different disciplines. Focusing predominantly on Bernese authors (names marked with asterisks), concepts which served as guidelines for research in these disciplines have been identified: 
- Natural philosophical anthropology was based partially on physiologically interpreted results of chemical investigation $\left(\right.$ Ith $\left.^{*}\right)$.

- Concepts in basic medical sciences:

In the tradition of Haller, chemical methods were used as dissection tools in anatomy to explain physiological functions (Gerber ${ }^{*}$, Aeby*).

Physiology got three methodical directions; one of them included chemical experiments (Purkynje, Valentin*).

Pathology was expected to become a physiological pathology; chemistry had to promote this transformation (Fueter *, Rokitansky, Virchow, Naunyn).

- Concepts of applied chemistry:

Urinary chemistry was used in diagnostics and to explain diseases physiologically (Parmentier and Déyeux, Ziegler ${ }^{*}$ ).

Relying on animal chemical methods, medical chemistry consolidated as physiological and pathological chemistry to explain healthy and pathological conditions of the human organism (Fourcroy, Nencki*).

Animal or organic chemistry as an independent science was applied to physiological and pathological problems (Brunner*, Liebig). There was also a physiological chemistry outside the framework of medical chemistry (Lehmann).

William Liebi

Stadt- und Universitätsbibliothek

Postfach 58

CH-3000 Bern 7 